\title{
Médiévales
}

Langues, Textes, Histoire

67 | automne 2014

Histoires de Bohême

\section{Entre idéal et polémique. La littérature politique dans la Bohême des Luxembourg}

Between Ideal and Polemic. Political Literature in Luxembourg Bohemia

Jan Vojtíšek et Václav Žůrek

\section{OpenEdition}

1 Journals

\section{Édition électronique}

URL : https://journals.openedition.org/medievales/7365

DOI : 10.4000/medievales.7365

ISSN : 1777-5892

\section{Éditeur}

Presses universitaires de Vincennes

\section{Édition imprimée}

Date de publication : 31 décembre 2014

Pagination : 11-30

ISBN : 978-2-84292-422-5

ISSN : 0751-2708

\section{Référence électronique}

Jan Vojtíšek et Václav Žưrek, «Entre idéal et polémique. La littérature politique dans la Bohême des Luxembourg », Médiévales [En ligne], 67 | automne 2014, mis en ligne le 31 décembre 2016, consulté le 23 avril 2022. URL : http://journals.openedition.org/medievales/7365 ; DOI : https://doi.org/10.4000/ medievales.7365 
Jan Vojtišek et Václav Žůrek

\section{Entre idéal et polémique La littérature politique dans la Bohême des Luxembourg}

Pendant le règne des Luxembourg en Bohême, la littérature politique a connu un grand développement. Parallèlement à la réception des modèles littéraires, une série de textes qui forment le champ de la littérature politique a pris naissance ${ }^{1}$. Le genre traditionnel des miroirs des princes, refondé sur de nouvelles bases grâce aux écrits d'Aristote, trouvait ses lecteurs avant tout parmi les savants de la cour royale: il constitue plutôt un mélange de différents textes, et sa délimitation comme genre au bas Moyen Âge demeure une vaste question ${ }^{2}$. À côté des traités théoriques sur l'organisation de la société et des miroirs des princes définissant l'idéal du gouvernement royal, d'autres textes appartiennent plutôt, à notre avis, à la catégorie de la littérature politique: des textes engagés qui s'efforcent de formuler un programme de parti dans le jeu politique, sans grand égard pour la forme littéraire.

En Bohême, comme dans l'Europe entière, on note une grande variété de moyens littéraires utilisés dans le discours politique. Au moment de l'avènement des Luxembourg au début du XIV ${ }^{\mathrm{e}}$ siècle, on peut compter, dans le genre de la littérature politique, la production des chroniqueurs engagés hors de la cour royale (Chronique rimée du soi-disant Dalimil, Chronique de Zbraslav) ${ }^{3}$. Ces derniers, après l'accession de Charles IV au

1. Cf. J.-P. Genet, La Genèse de l'État moderne. Culture et société politique en Angleterre, Paris, 2003, p. 292-305.

2. Cf. W.Berges, Die Fürstenspiegel des hohen und späten Mittelalters, Stuttgart, 1938; J.-P. GEnet éd., Four English Political Tracts of the Later Middle Ages, Londres, 1977, p. IX-XIX; J. KRYNEN, Idéal du prince et pouvoir royal en France à la fin du Moyen Âge (13801440). Étude de la littérature politique du temps, Paris, 1981 ; C. J.NEDERMAN, «The Mirror Crack'd: The Speculum principum as Political and Social Criticism in the Late Middle Ages », The European Legacy, 3 (1998), p. 18-38; J. Blanchard et J.-C.MüHLETHALer, Écriture et pouvoir à l'aube des temps modernes, Paris, 2002, p. 7-32.

3. Cf. Staročeská kronika tak řečeného Dalimila, éd. J. DaňHelka, K. НÁdeK, B. HaVRÁNEK et N.KvítKová, Prague, 1988 (2 vol.); $c f$. aussi la traduction française préparée 
trône, se dirigent vers la cour et, sous le contrôle du pouvoir royal, adoptent incontestablement une position favorable au roi. La fondation de l'Université à Prague contribue aussi à faire de la cour de Charles IV un centre culturel et intellectuel. Le concept idéologique clair de l'empereur est exprimé par les œuvres liées à la personne du souverain: son autobiographie Vita Caroli, le recueil de moralités Moralitates, ou un miroir de prince anonyme de la fin du règne de Charles $\mathrm{IV}^{4}$.

La littérature politique en Bohême était aussi façonnée par une lecture des œuvres fondamentales comme le Policraticus de Jean de Salisbury, le De regimine principum de Gilles de Rome, l'œuvre de Thomas d'Aquin ou encore le Secretum secretorum, ce qui est attesté par des manuscrits bien conservés. Plusieurs de ces textes, mettant fortement l'accent sur la morale, avaient été traduits en tchèque à la fin du XIv ${ }^{\mathrm{e}}$ siècle (par exemple Le Livre du jeu des échecs de Jacques de Cessolesou le Breviloquiumde virtutibus antiquorum de Jean de Galles ${ }^{5}$ ). Cette dimension moralisatrice est typique de la seconde moitié du XIV ${ }^{\mathrm{e}}$ siècle. Les auteurs appartiennent de moins en moins à l'élite intellectuelle de la cour. Ils s'adressent à la société politique au sens le plus large du mot, au moyen de traités et de poèmes allégoriques. Outre leur caractère moral, leurs œuvres ont une fonction didactique et, surtout, religieuse. Ce développement coïncide avec la crise du pouvoir royal, notamment sous le règne de Venceslas IV.

L'engagement croissant des œuvres, composées dans le contexte de la polémique avec le pouvoir royal, sur le rôle de la haute noblesse dans le gouvernement du royaume, dont le meilleur exemple est le Nouveau conseil de Smil Flaška de Pardubice, culmine après 1420 dans une série des polémiques interconfessionnelles entre hussites et catholiques ${ }^{6}$. Il importe de mentionner que la plupart de ces textes sont écrits en tchèque, ce qui ne signifie pas seulement un cercle grandissant de destinataires, mais aussi que l'on trouve parmi les auteurs un nombre croissant de nobles ayant reçu une éducation universitaire.

Le champ de la littérature politique n'est pas seulement défini par son contenu, c'est-à-dire par les différents ouvrages, mais aussi et

par É. AdDE (à paraître), et le Chronicon Aulae Regiae, éd. J. EmLER, FRB IV, Prague, 1884, p. 1-337.

4. Cf. Vie de Charles IV de Luxembourg, éd. P. Monnet et J.-C. Sснмiтt, Paris, 2010 ; Moralitates Caroli quarti imperatoris, éd. K. WоткE, Zeitschrift des deutschen Vereines für die Geschichte Mährens und Schlesiens, 1 (1897), p. 59-73; Ein Fürstenspiegel Karls IV, die Geschichte Mährens und Schl

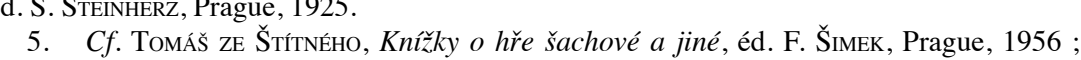
Staročeský překlad spisu Jana Guallensis O čtyřech stěžejných ctnostech, éd. F. ŠIMEK, Prague, 1952.

6. Cf. F. M. Bartoš, «Z politické literatury doby husitské», Sborník historický, 5 (1957), p. 21-70; V. Herold, I. Müller et A. HavLí̌̌́, Politické myšleni pozdního středověku a reformace, Prague, 2011, p. 118-372. principalement par son auditoire: le destinataire forme en même temps l'environnement dans lequel l'œuvre est écrite et celui pour lequel elle est rédigée, et ce destinataire, c'est la société politique. Nous sommes ici inspirés par le modèle développé par John Bell Henneman pour la France du bas Moyen Âge ${ }^{7}$. On définira ainsi la société politique comme celle des hommes qui exercent leur pouvoir politique ou possèdent la capacité d'intervenir dans la décision politique, soit au niveau suprême, à la cour et dans les institutions de gouvernement, soit à des niveaux inférieurs, c'est-àdire dans les pouvoirs locaux.

Dans les deux analyses qui suivent, nous souhaitons démontrer la corrélation entre littérature et société politique dans la Bohême du XIV ${ }^{\mathrm{e}}$ siècle. Tandis que, sous le règne de Charles IV, l'idéal du roi fut bien décrit dans plusieurs ouvrages, le règne de son fils Venceslas IV est caractérisé par l'affaiblissement du pouvoir royal qui culmine en une querelle entre le roi et les nobles du royaume. Nous présenterons ainsi les deux facettes du fonctionnement de la littérature politique: la définition de l'idéal et la polémique avec le pouvoir royal.

\section{La sagesse et son importance pour l'image du souverain dans les miroirs du prince en Bohême ${ }^{8}$}

Charles IV et son entourage accordent une grande attention à la représentation de la majesté du souverain. Parmi les aspects les plus flagrants, on trouve au premier lieu la piété, omniprésente dans l'image contemporaine de Charles IV : il collectionne les reliques, fonde des autels et soutient l'Église par tous les moyens ${ }^{9}$. Charles est par ailleurs assez souvent représenté sous les traits du roi sage (rex sapiens). À l'instar de Salomon, modèle biblique, la sagesse caractérise le souverain doté de capacités intellectuelles et de sens politique, qui sait utiliser ces facultés dans son gouvernement, qui est un homme savant et intelligent et qui mène une politique avisée ${ }^{10}$

7. Cf. J. B. Henneman, Olivier de Clisson et la société politique française sous les règnes de Charles V et Charles VI, Rennes, 2011, p. 19-20, qui s'inspire des thèses de R. CAZEUES, La Société politique et la crise de la royauté sous Philippe de Valois, Paris, 1958.

8. Cette première partie est rédigée par Václav Žůrek. Cette recherche a bénéficié du financement du Conseil européen de la recherche au titre du Programme de la Communauté européenne, septième programme-cadre (FP7/2007-2013)/ERC, conventions de subvention $\mathrm{n}^{\circ} 263672$

9. Pour son style de gouvernement, voir le livre récent de M.BAUCH, Auserwählung - Frömmigkeit - Heilsvermittlung. Zur Herrschaftspraxis Kaiser Karls IV., Vienne-CologneWeimar, 2014.

10. Cf.J.-P.BoudET, «Le modèle du roi sage aux XIII et XIV siècles : Salomon, Alphonse $\mathrm{X}$ et Charles V», Revue historique, 647 (2008), p. 545-566. 
Le roi sage n'est pas seulement un idéal littéraire : plusieurs souverains de l'Europe médiévale furent considérés comme sages et même appelés par ce surnom, comme Alphonse X de Castille (el Sabio), Robert I Ir de Naples (il Saggio), ou encore Charles V de Valois (le Sage), neveu de Charles IV de Luxembourg ${ }^{11}$. Malgré la diversité géographique et culturelle, l'exemple de ces sages souverains nous permet de définir, bien que de façon rudimentaire, quels qualités personnelles et actes de gouvernement caractérisent le roi sage. En effet, bien que l'idéal change avec le temps, il reste assez stable au cours du XIII et du XIV ${ }^{\mathrm{e}}$ siècle $^{12}$. Il se décline surtout en quatre figures: le roi législateur, le roi mécène, le roi lecteur et le roi auteur. Toutes ces catégories se retrouvent dans l'image de Charles IV de Luxembourg qui, même s'il ne porta jamais le surnom de «sage», était déjà considéré comme tel par ses contemporains ${ }^{13}$.

L'activité législative répond bien à la vision d'un souverain célébré pour sa sagesse : pour Eva Schlotheuber, c'est exactement cette dimension de son règne et les grands codes de lois qui font que Charles IV mérite le titre de roi sage ${ }^{14}$. À côté du patronage artistique à la cour de Prague, il faut surtout souligner le soutien à la production d'une littérature vernaculaire, ce qui vaut d'ailleurs pour Charles IV autant que pour les autres rois mentionnés. L'essor de la littérature en langue vulgaire est souvent lié à la floraison culturelle, ce qui contribue à renforcer l'image. Un autre aspect important présent chez les rois sages est le rôle de fondateur et de protecteur de l'Université: Charles IV fonda en 1348 à Prague la première Université au nord des Alpes et à l'est du Rhin, sanctionnant par ailleurs, en tant qu'empereur, la fondation ou la confirmation de plusieurs autres studia generalia en Europe (Pavie, Sienne, Orange, etc.) ${ }^{15}$. Mentionnons aussi sa prédilection pour les livres, qui témoigne de la culture savante de ce roi. Certains rois, comme Charles V en France, collectionnaient des livres, commandaient des manuscrits richement enluminés et sont souvent

11. Sur Alphonse X (1252-1284), voir R. BunNs éd., Emperor of Culture: Alfonso X the Learned of Castile and His Thirteenth-Century Renaissance, Philadelphie, 1990. Sur Rober $\mathrm{I}^{\text {er }}$ de Naples (1309-1343), voir S. KelLy, The New Solomon: Robert of Naples (1309-1343) and Fourteenth-Century Kingship, Leyde - Boston, 2003. Sur Charles V (1364-1380), voir notamment F.Autrand, Charles V, le Sage, Paris, 1994.

12. $C f$. J.-P. Boudet, «Le modèle du roi...» ; M. Chopin-PAGotto, «La prudence dans les Miroirs du prince», Chroniques italiennes, 60 (1999), p. 87-98.

13. Cf. V.Ž ŽREK, «Karl IV. als der weise Herrscher», dans Heilige, Helden, Wüteriche. Verflochtene Herrschaftsstile im langen Jahrhundert der Luxemburger (sous presse).

14. Е. Sснцотнеuber, «Der weise König. Herrschaftskonzeption und Vermittlungsstrategien Kaiser Karls IV. (1378)», Hémecht. Zeitschrift für Luxemburger Geschichte, 63 (2011), p. 265-279.

15. $C f$. M.Kubová, «Univerzity založené Karlem IV. Obraz o zakladatelské činnosti universitní císaře Karla IV.», Acta Universitatis Carolinae - Historia Universitatis Carolinae Pragensis, 11 (1970), p. 7-31. représentés dans les scènes de dédicace ${ }^{16}$. Charles IV utilise plutôt le livre comme un medium privilégié, commandant de nombreux ouvrages rédigés pour lui par les membres de la cour ou par des savants étrangers. Le soin porté à la production historique, théologique ou juridique, lui a valu la réputation de souverain savant, et la paternité de certains ouvrages latins (l'autobiographie, les Moralitates, la Légende de saint Venceslas) y contribue encore plus. Cela vaut à Charles le titre, rare parmi les rois du XIII ${ }^{\mathrm{e}}$ et du XIV ${ }^{\mathrm{e}}$ siècle, de roi lettré (rex litteratus) ${ }^{17}$

Charles IV ne manque aucune occasion de se présenter en tant que savant sur le trône. Dans son autobiographie, qui est déjà en elle-même la preuve de son extraordinaire instruction, il raconte qu'à la cour de France, où il passa sept années de son enfance (1323-1330), son précepteur lui apprit «les rudiments d'éducation lettrée ${ }^{18}$ ». Il souligne la valeur de ces compétences et rappelle que le roi Charles IV le Bel, qui le fit instruire, était lui-même «ignorant des lettres ${ }^{19}{ }$. Le chroniqueur Beneš Krabice de Weitmile raconte même que, pendant son séjour à Paris, le jeune Charles étudia à l'Université ${ }^{20}$. Il faut plutôt lire ce passage comme une affirmation $\mathrm{du}$ caractère extraordinaire de son éducation. La réputation de Charles IV comme roi sage et savant n'était pas un caprice personnel: au contraire, elle résulte d'une représentation systématique et consciente de l'empereur, à la fois au sein du milieu de sa cour et devant les yeux des ambassadeurs étrangers. À travers cette représentation, Charles IV se positionne aussi par rapport au type du roi-chevalier, représenté par son père Jean l'Aveugle, et insiste plutôt sur la diplomatie et la prudence politique ${ }^{21}$.

$\mathrm{Du}$ succès de cette politique de représentation témoignent non seulement les auteurs de la cour praguoise, mais aussi plusieurs auteurs étrangers ${ }^{22}$. En guise d'exemple, nous citerons l'enthousiasme de Guillaume de Machaut, ancien secrétaire de Jean l'Aveugle, dans son poème La Prise

16. $C f$. C. R. Sherman, «Representations of Charles V of France (1338-1380) as a Wise Ruler», Medievalia et Humanistica, n. s.2 (1971), p. 83-96.

17. Cf. A. Vidmanová, «Karel IV. jako spisovatel», dans Karel IV. Literární dílo, Prague, 2000, p. 9-22 ; F. RÄDLE, «Karl IV. als lateinischer Autor», dans F. SEIBT éd., Kaiser Karl IV. Staatsmann und Mäzen, Munich, 1978, p. 253-260.

18. Vie de Charles IV, éd. P. MonNET et J.-C. SснмiтT, p. 20-21.

19. Ibid.

20. Beneš Krabice de Weitmile, Cronica ecclesiae Pragensis, éd. J. EmLer, FRB IV, Prague, 1884, p. 459-548, ici p. 517.

21. Cf. M. Margue éd., Un itinéraire européen. Jean l'Aveugle, comte de Luxembourg et roi de Bohême 1296-1346, Bruxelles, 1996.

22. Cf. H. Herкомmer, «Kritik und Panegyrik. Zum literarischen Bild Karls IV», Rheinische Vierteljahrsblätter, 44 (1980), p. 68-116. 
d'Alixandre, où il décrit l'accueil fait par Charles IV à son maître d'alors, Pierre de Lusignan ${ }^{23}$ :

\section{On pourroit en nulle terre}

nul plus sage homme de li querre

con dit ca et dela mons

que cest li secons salemons.

Même les chroniqueurs français font écho à cette réputation du roi sage qui préfère la fine diplomatie à la force. L'auteur de la Chronique des quatre premiers Valois s'exprime ainsi sur Charles IV : «Et fut cestui empereur ung tres grant sages homs et conquist plus l'empire par sens que par armes ${ }^{24}$.»

Toutefois, ce sont les auteurs écrivant à la cour de Prague qui contribuent le plus à la construction de la figure de Charles comme roi sage. Les œuvres qui transmettent cette idée sont assez diverses: mentionnons pour l'exemple un poème et un éloge funèbre.

Le poète allemand Henri de Mügeln, invité à la cour de Prague, composa en allemand dans les années 1350 un poème allégorique Der Meide Kranz (La couronne de la Vierge), dans lequel il décrit la querelle savante entre les arts libéraux. Dans la deuxième partie du poème, les vertus entrent en scène et l'une des vertus cardinales, la Sagesse personnifiée, s'adresse à Charles IV pour lui dire combien elle est importante pour le souverain: «Prince, tu dois être le miroir de tes sujets, ils se voient dans toi. Comportetoi alors prudemment, car Dieu t'a élevé au-dessus d'eux, c'est pourquoi tu dois être beaucoup plus sage qu'eux ${ }^{25}$.»

Une autre façon de construire la gloire de Charles comme roi sage est entreprise par l'éloge prononcé en public, à l'occasion des obsèques du roi, par l'archevêque de Prague, Jean de Jenstein, qui insiste sur plusieurs vertus de l'empereur mort, notamment ses compétences intellectuelles. D'après lui, Charles avait l'esprit de la sagesse (spiritus sapiencie) et il était plus sage que Salomon, parce qu'il savait des choses que Salomon ne savait pas. Charles IV possédait aussi l'esprit intellectus et l'esprit de la science (spiritus sciencie), par lequel Jean désigne ses connaissances théologiques et sa capacité d'exposer et de commenter les Psaumes ou l'Évangile et d'en

23. Gullaume de Machaut, La Prise d'Alixandre, éd. R. B. Palmer, New York-Londres, 2002, p. 80

24. Chronique des quatre premiers Valois (1327-1393), éd. S. LucE, Paris, 1861, p. 278.

25. Henri de Mügeln, Der Meide Kranz, dans K.Stackmann éd., Kleinere Dichtungen Heinrichs von Mügeln, t. 2, Berlin, 2003, p. 140: «du fürste, salt ein spigel sin/in tat den underseßen din,/das sie beschouwen sich in dir: [...] dir, fürste, zimet wise tat:/recht als dich got erhaben hat/übr ander die genoßen din./also vil saltu wiser $\sin »$. discuter avec les maîtres universitaires ${ }^{26}$. L'archevêque mentionne aussi les compétences linguistiques de Charles, qui parlait cinq langues (tchèque, allemand, français, italien et latin). Cette réputation de polyglotte devient aussi une dimension importante de son image de roi savant.

Dans le discours littéraire de la cour de Charles IV, la sagesse du prince devient ainsi, à côté de la piété, la qualité la plus importante de l'image du prince idéal. Il est donc naturel que cet idéal du prince, formulé dans le milieu de la cour, influence aussi l'ensemble de la littérature politique désormais composée dans la Bohême des Luxembourg. Dans cette vaste catégorie, ce sont surtout les miroirs des princes qui nous intéresseront. Ils déterminent non seulement la bonne forme de gouvernement, mais formulent aussi des exigences quant à la personnalité du prince, conformément à l'idée, répandue au Moyen Âge, selon laquelle le bon gouvernement repose sur un roi sage. Trois miroirs des princes rédigés dans la deuxième moitié du $x v^{\mathrm{e}}$ siècle prouvent l'importance et la primauté de la sagesse parm les vertus nécessaires du bon roi. Ils furent pourtant composés dans des contextes assez différents: l'un à la cour de Charles IV, le second dans le milieu ecclésiastique de Prague, le troisième (rédigé en tchèque à la différence des deux précédents qui sont en latin) dans le milieu nobiliaire.

Le premier miroir latin, écrit en 1377, prend la forme de deux lettres. Depuis son édition en 1925, il est considéré comme une lettre de Venceslas IV et la réponse de Charles IV ${ }^{27}$. Dans la première lettre, très courte, le fils, le roi récemment élu, demande à son père - l'empereur - de lui donner des conseils sur la manière de bien gouverner le royaume. Le texte central consiste en la réponse de l'empereur, représentée par un long traité moral, assez général et sans références concrètes à la vie des Luxembourg ou de l'Europe centrale au XIV ${ }^{\mathrm{e}}$ siècle. La question de l'auteur n'est pas encore résolue; à cause des nombreuses citations d'auteurs antiques, il est habituellement situé parmi les savants italiens de la cour de Charles IV : on pense en particulier à Nicolo Beccari, le précepteur de ses fils ${ }^{28}$.

Ce texte est une sorte de catalogue des vertus requises pour un prince, commenté par l'empereur. Il semble que le texte ait été écrit directement pour le jeune Venceslas, ce que révèlent plusieurs passages. Ainsi le père insiste sur la nécessité de la tempérance et de la clémence et sur le fait qu'un bon roi ne doit jamais succomber aux attaques de rage; or la réputation colérique de Venceslas n'est plus à faire. Charles IV était persuadé que le prince pouvait apprendre l'art de gouverner, ce dont témoigne surtout son autobiographie, la Vita Caroli, dédiée «à ceux qui siègeront après moi sur

26. Sermo factus per dominum Johannem archiepiscopum Pragensem post mortem imperatoris Caroli IV, éd. J. EMLER, FRB III, Prague, 1882, p. 427.

27. Ein Fürstenspiegel Karls IV., éd. S. STEINHERZ, Prague, 1925, p. 8-9.

28. Cf. J.Ludví́KovsKÝ, «Anonymní zrcadlo knížecí připisované Karlu IV.», Studie o rukopisech, 14 (1975), p. 125-127. 
mon double trône ${ }^{29}{ }^{\gg}$. Le savoir-faire du métier de roi est aussi décrit dans ce miroir de l'an 1377 sous la forme d'une lettre de l'empereur ${ }^{30}$. L'auteurmoraliste inconnu mentionne la sagesse et la prudence comme les vertus nécessaires pour le roi et souligne, en citant la Bible (surtout les Proverbes), que le bon souverain apprécie la sagesse bien plus que l'or, l'argent ou les perles. Il revient plusieurs fois sur cette vertu pour souligner son importance. La présentation de la sagesse et de la raison dans ce texte correspond bien à l'image de Charles IV tel qu'il apparaît dans la production littéraire de sa cour.

La littérature politique ne naît toutefois pas seulement à la cour. Le moine Michel, vicaire de la chartreuse de Prague, est l'auteur, en 1387, du De regimine principum seu de quatuor virtutibus cardinalibus pro eruditione principum libri IV $V^{31}$. Michel l'a écrit, d'après l'expression qu'il emploie dans le prologue, pour «Robert le jeune, duc Bavière» (Ropertus junior, dux Bavariae), de la famille de Wittelsbach ${ }^{32}$. Bien qu'on ne puisse pas déterminer qui était le destinataire - le prochain roi des Romains (1410) ou son père homonyme (1398) -, il est intéressant de noter que le clerc de Prague donne des conseils au prince de la famille concurrente des Luxembourg. Michel rédige son miroir sous la forme d'un dialogue entre le jeune $\mathrm{R}$ (obert), posant les questions, et $\mathrm{M}$ (ichael), répondant à ces questions d'une façon très claire et structurée. Comme la plupart des œuvres du genre des miroirs, ce texte a souffert du mépris des historiens, qui le considéraient comme manquant d'originalité; il est vrai que Michel de Prague a repris la plupart de ses conseils chez des classiques du genre (Gilles de Rome, Thomas d'Aquin, Peraldus ou le Pseudo-Sénèque) et qu'il cite fréquemment ses autorités ${ }^{33}$

L'ouvrage de Michel est divisé en quatre livres, dont chacun est consacré à une vertu cardinale: la prudence, la modération, la justice et la force (prudencia, temperancia, iusticia et fortitudo). L'auteur suit le système classique, mais il place la prudence à la première place et fait aussi comprendre, par son exposé, que la prudence est la vertu essentielle du bon

29. Vie de Charles IV, éd. P. Monnet et J.-C.SchmitT, p. 3

30. $C f$. M.NeJEDLÝ, «L'idéal du roi en Bohême à la fin du XIV siècle. Remarques sur le Nouveau conseil de Smil Flaška de Pardubice», dans D. Boutet et J.Verger éd., Penser le pouvoir au Moyen Âge. Etudes offertes à Françoise Autrand, Paris, 2000, p. 247-260 (p. 248249).

31. The "De quatuor virtutibus cardinalibus pro eruditione principum" of Michael the Carthusian of Prague: The Critical Text and Study, éd. W. G. SToRey, Salzbourg, 1972 (Analecta Cartusiana, 6). W. G.Storey n'édite que le prologue et le premier livre. Les trois derniers livres sont publiés dans Michael of Prague, De quatuor virtutibus cardinalibus pro eruditione principum (Book II - IV), éd. R. Witкowski, Salzbourg, 2009 (Analecta Cartusiana, 6/2)

32. The "De quatuor virtutibus...", p. 110

33. $C f$. l'analyse très riche des auteurs cités et utilisés, ibid., p. 37-56. prince. La prudence et la sagesse - car chez Michel les deux notions son très proches - sont très appréciées dans l'idéal du prince construit par le miroir. Ce dernier décrit la sapience comme une vertu, non pas innée, mais qui est le résultat de l'expérience et de l'instruction (sur laquelle il met aussi l'accent) $)^{34}$.

L'appréciation du système des vertus n'est pas uniforme dans ce miroir: pour Michael Hohlstein, au centre de ce texte se trouve la vertu de clémence (clementia) qui, pour Michel, est la plus importante parce que le souverain peut grâce à elle éviter de devenir un tyran ${ }^{35}$. Il est par ailleurs probable que Michel se positionne ici contre le roi Venceslas IV, renommé pour sa fureur incontrôlée. Malgré sa distance relative de la cour, Michel est au fait de la situation politique dans le royaume de Bohême et dans l'Empire, ce dont témoigne, dans le prologue, sa complainte sur le temps, quand les vertus sont expulsées du pays ${ }^{36}$. Il utilise de toute évidence Venceslas IV comme un modèle négatif du mauvais roi ${ }^{37}$. Son ouvrage est très moralisateur, le miroir se consacrant beaucoup plus à la dimension morale du personnage du roi qu' à la pratique du gouvernement ${ }^{38}$. Le moment important, dans le premier livre sur la prudence, est le besoin de bon conseil : d'après Michel, le prince doit avoir un conseil, et il explique aussi dans quels cas il doit le consulter ${ }^{39}$. Le roi doit aussi chercher la sagesse chez ses conseillers. Michel mentionne trois manières par lesquelles le prince doit utiliser la sagesse dans son gouvernement : en cœur, en parole et en action ${ }^{40}$ La conception de la sagesse et de la morale princière de Michel de Prague correspond bien à la tradition de la cour de Charles IV.

L'influence de cette conception s'observe aussi dans un texte assez différent: le miroir de prince Nová rada (Nouveau conseil) de Smil Flaška de Pardubice, écrit en langue vernaculaire ${ }^{41}$. La sagesse y apparaît assez souvent, et elle est plusieurs fois évoquée en tant que vertu indispensable au

34. Pour l'édition du livre sur la prudence, voir ibid., p. 121 sqq., et le commentaire de l'éditeur, p. 60-74. $C f$. aussi E. HerrmanN, «Der Fürstenspiegel des Michael von Prag», Historisches Jahrbuch,91 (1971), p. 22-45 (p. 42).

35. Cf. M. Hohlsters, «Clemens princeps: Clementia as a Princely Virtue in Michael of Prague's De regimine principum», dans I. P. BejcZY et C. J. Nederman éd., Princely Virtues in the Middle Ages. 1200-1500, Turnhout, 2007, p. 201-217, ici p. 210-217.

36. The "De quatuor virtutibus...", p. 111.

37. Cf. P. Rychterová, «"Hör zu, König, der du meinen Rat verlangst!" Das richtige Regiment in der alttschechischen Literatur der zweiten Hälfte des 14. Jahrhunderts», dans G. Briguglia et T.RickLin éd., Thinking Politics in the Vernacular from Middle Ages to the Renaissance, Fribourg, 2011, p. 129-149.

38. Cf. W.IWANCZAK, «Der Kartäuser Michael aus Prag - ein Moralist des späten Mittelalters», dans S.LORENZ éd., Bücher, Bibliotheken und Schriftkultur der Kartäuser Festgabe zum 65. Geburtstag von Edward Potkowski, Stuttgart, 2002, p. 83-92.

39. The "De quatuor virtutibus cardinalibus...", p. 152-157.

40. Ibid., p. 115

41. Pour la présentation détaillée de ce poème voir infra. 
bon roi. Par rapport aux ouvrages précédents, la perspective de l'auteur est pourtant assez différente. Smil insiste, tout comme Michel de Prague, sur la nécessité d'utiliser le conseil et sur le fait qu'il faut y avoir des hommes sages. Provenant lui-même d'une famille noble, il souligne l'utilité de la coopération entre le roi et la noblesse et il exige, en conséquence, les mêmes vertus (principalement la sagesse ou justice) du roi et de ses conseillers: «Le roi sage doit avoir des sages hommes dans son conseil et ils doivent se rendre utiles ${ }^{42}$.» Le roi est alors obligé de s'entourer d'hommes bien choisis et dotés des vertus exigées. On peut voir dans ce passage un motif présent déjà chez Charles IV : le bon roi s'entoure de bons conseillers. La sagesse du roi se manifeste aussi dans sa capacité à choisir prudemment ses collaborateurs.

\section{La société politique dans le Nouveau conseil de Smil Flaška de Pardubice ${ }^{43}$}

Roi lion, il était une fois,

a envoyé des messagers en maintes qualités,

dans toutes les régions et de tous les côtés,

chez ses comtes et ses seigneurs,

chez les animaux grands et petits,

afin que tous devant lui se réunissent.

Ainsi commence le célèbre poème médiéval tchèque du Nouveau conseil, composé par Smil Flaška de Pardubice (1403), issu d'une famille de la haute noblesse tchèque qui s'était élevée au service des rois de la dynastie de Luxembourg ${ }^{44}$. Deux ecclésiastiques, l'archevêque de Prague Ernest de Pardubice (1364) et le chanoine Bohuš, oncles de Smil Flaška, avaient assuré à leur jeune neveu une instruction exceptionnelle, imprimant

42. Smil FlašKa de Pardubice, Nová rada, v. 477-478, éd.J. Dañhelka, Prague, 1950, p. 30-31 (désormais Nová rada).

43. Cette seconde partie est rédigée par Jan Vojtî̌ek. Cette étude a été réalisée dans le cadre du projet $\mathrm{n}^{\circ} 24213$, «La perception des différences nationales en bas Moyen Âge : les pays tchèques comme un exemplum?», soutenu par l'Agence de la recherche scientifique de pays tchèques comme un exemplum ?»,
l'Université Charles de Prague (GAUK).

44. Nová rada, v. 1-6. La traduction de kniežata par «comtes» n'est pas littérale mais
'Université Charles de Prague (GAUK). elle reflète mieux la réalité de la Bohême médiévale. Les études de base sont : J. B. ČAPEK, «Alegorie Nové rady a Theriobulie», Věstník Královské české společnosti nauk, 1937, p. 1-53 ; J. B. ČAPEK, «Vznik a funkce Nové rady», Věstník Královské české společnosti nauk, 1938, p. 3-100 ; en français, voir M. NeJEDLÝ, «L'idéal du roi en Bohême à la fin du XIV siècle...», p. 247-260. une double marque sur sa personnalité et ses œuvres: une religiosité profonde et une confiance en l'idéologie de la cour de Charles IV ${ }^{45}$.

Son instruction et la situation politique du royaume de Bohême ont incité Smil à composer le Nouveau conseil. Ce poème s'inscrit dans la tradition des textes allégoriques critiques et politiquement engagés, composés en langue vernaculaire, et participe d'une tendance qui se manifeste partout en Europe occidentale au cours du XIV ${ }^{\mathrm{e}}$ siècle ${ }^{46}$. Le jeune roi lion convoque ses sujets animaux afin qu'ils lui accordent leur conseil sur les qualités d'un bon roi et sur le bon gouvernement. Les animaux se succèdent tour à tour, un oiseau et un quadrupède, et proposent de bons mais aussi de mauvais conseils qui contiennent un degré significatif d'ironie ${ }^{47}$. L'auteur utilise ce moyen littéraire pour émettre une critique sévère du roi Venceslas IV de Bohême et de ses familiers. Le concept fait immédiatement penser au Roman de Fauvel du début du XIve siècle, où l'allégorie animale véhicule une critique politique et sociale de l'entourage du souverain ${ }^{48}$.

Il serait néanmoins erroné de voir le Nouveau conseil comme un texte exclusivement critique. Si l'ironie joue un rôle important, la visée principale de l'œuvre reste de renseigner le roi sur le bon gouvernemen à travers la mise en relief de ses fautes: la dimension positive demeure toujours la plus forte. On peut ainsi considérer le Nouveau conseil comme un miroir de prince sous forme allégorique. Cette composition s'apparente à certaines autres œuvres écrites pendant la seconde moitié du XIV siècle. L'historiographie tchèque s'est efforcée de trouver des modèles dans d'autres littératures de l'Europe médiévale, surtout anglaise (The Parlement of Fowles de Geoffrey Chaucer ${ }^{49}$ ) et française (la Fiction d'Aigle d'Eustache Deschamps et les œuvres de la plume de son maître, poète de la cour de Jean l'Aveugle, Guillaume de Machaut ${ }^{50}$ ). Les mêmes schémas structurants et narratifs encadrent des similitudes et corrélations de loci communes dans la peinture de l'idéal royal. Ce type de littérature connaît par ailleurs, à

45. Smil Flaška aurait obtenu le titre de bachelier à l'université de Prague et il pouvait profiter de la bibliothèque de ses parents (et d'autres intellectuels de la cour, comme le chancelier Jean de Středa): voir M. NeJEdLÝ, «L'idéal du roi en Bohême...», p. 251-252. Sur la maison de Pardubice et l'archevêque Ernest, voir F. ŠEBEK, «Arnošt z Pardubic a jeho rod», dans L. Bobková, R. GŁadriewicz et P. Vorel éd., Arnošt z Pardubic (1297-1364). Osobnost - okruh - dédictví, Wroclaw - Prague - Pardubice, 2005, p. 83-91, et Z HuEDíkoví, Arnoš z Pardubic. Arcibiskup, zakladatel, rádce, Prague, 2008.

46. $C f$. J. BLANChARD et J.-C. MühLETHALER, Écriture et pouvoir...

47. Cf. J. B. ČAPEK, «Die Ironie des Smil Flaška», Slavische Rundschau, 10 (1936), p. 68-79.

48. Roman de Fauvel, éd. A. Strubel, Paris, 2012.

49. Cf. A. Kraus, «K Smilově "Nové radě”», Listy filologické, 31 (1904), p. 199-212.

50. Cuvres complètes d'Eustache Deschamps VI, bal. 1589, v. 1-604, éd. M. DE QueuX De SaINT-Hilaire, Paris, 1889, p. 147-167 ; P. M. HašKovec, «Některá themata literatur západních v českém písemnictví II», Listy filologické, 44 (1917), p. 252-269. 
cette époque, une popularité croissante dans l'Europe entière. Dans l'espace allemand, qui était bien sûr culturellement et politiquement le plus proche de la Bohême, on trouve ainsi une série de conseils d'oiseaux allemands ${ }^{51}$. Le Nouveau conseil se prête non seulement à une étude de la filiation et des sources d'inspiration, mais aussi à des analyses portant sur les genres et les moyens littéraires mis en œuvre. En outre, il rend compte du développement de l'État, de ses structures et de ses institutions à la fin du Moyen Âge, et reflète les jeux politiques liés à l'évolution du royaume.

On suivra cette piste des jeux politiques et du développement des institutions de pouvoir dans la Bohême pré-hussite. Le poème est conservé dans une version qui représente très probablement une seconde rédaction datant de $1394^{52}$. Cette année est marquée par un conflit grave entre le roi et un parti de la haute noblesse, rassemblé dans une «Union des seigneurs» soutenue par le cousin du roi, le margrave Jošt de Moravie. Les signes de la crise étaient déjà apparus au cours des années précédentes, avec des frictions entre le roi Venceslas et l'Église, qui culminèrent en 1393. À la fin de cette année, Venceslas survécut à une tentative d'empoisonnement: apparut alors la question de la succession d'un roi sans enfants, qui fit s'activer ses parents. Le 8 mai 1394, le roi fut arrêté à Králův Dvůr par les chefs de l'Union des seigneurs, le grand burgrave de Prague Ota de Bergov et Jindřich de Rožmberk. Il fut accusé de nombreux méfaits et emprisonné d'abord à Prague, puis dans le domaine de la maison de Rožmberk, en Bohême du Sud, et chez leurs alliés en Autriche. Le duc Jean de Görlitz, frère du roi, ouvrit immédiatement les hostilités contre l'Union et obtint la conclusion d'un accord stipulant la libération du roi au début d'août 1394 Les seigneurs revendiquaient un rôle essentiel dans le conseil royal et d'autres offices clés, au détriment des «mignons» issus des couches inférieures que les seigneurs accusaient du mauvais état du royaume ${ }^{53}$. Jean de Görlitz accepta ces conditions mais Venceslas, une fois libéré, ne voulut pas les ratifier. Pendant les deux années suivantes, la situation oscilla entre guerre et négociations, jusqu'au printemps 1396, où l'arbitrage de Sigismond, frère du roi et roi de Hongrie, mit provisoirement fin aux

51. Cf. J. B. ČAPEK, «Vznik a funkce Nové rady...», p. 90-93

52. Ibid., p. 3-100

53. Sur la composition du conseil royal et de la cour, voir I. HLAVÁČEK, Das Urkundenund Kanzleiwesen des böhmischen und römischen Königs Wenzel (IV.) 1376-1419. Ein Beitrag und Kanzleiwesendes büher

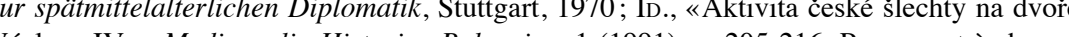
Václava IV.», Mediaevalia Historica Bohemica, 1 (1991), p. 205-216. Pour une très bonne analyse quantitative montrant une étonnante petite proportion de la petite noblesse dans la cour de Venceslas IV comparée aux autres princes de l'époque (y compris Charles IV), avec une réflexion sur la «culpabilité des parvenus» comme un topos universel, voir R. NovOTNÝ, «Ráj milců ? Nižší šlechta na dvoře Václava IV.», dans D. DvořáčKová-MaLÁ et J. ZelenKA éd., Dvory a rezidence ve středověku II. Skladba a kultura dvorské společnosti, Prague, 2008, p. 215-229. désordres. C'est pendant cette période que Smil Flaška de Pardubice, déçu par un long procès perdu au sujet de l'échute de la seigneurie de Pardubice et ayant des liens économiques avec les membres de l'Union, rejoignit le parti des seigneurs. Après l'arbitrage du roi Sigismond favorable aux seigneurs, Smil Flaška tint l'un des quatre offices les plus importants du pays, celui de grand scribe du royaume. Mais le roi Venceslas n'accepta pas sa défaite et la situation resta tendue jusqu'à son second emprisonnemen en 1402-1403, cette fois-ci opéré par son frère, l'ambitieux roi Sigismond. C'est alors que le poète Smil Flaška de Pardubice perdit la vie dans une escarmouche contre les combattants royaux, près de la ville de Kutná Hora, le 13 août $1403^{54}$

Voilà pour le décor et les acteurs des jeux politiques contemporains de la rédaction du Nouveau conseil. Quelques moments du résumé historique révèlent déjà des tendances de l'œuvre. Issu de la haute noblesse et politiquement engagé, Smil Flaška adopte une position conforme à son milieu social et à ses aspirations. Quelle est donc sa vision de la hiérarchie sociale et de la société politique? Quelle institution gouvernementale lui sert de cadre pour décrire la société politique et quel rôle lui assigne-t-il?

Le lion représente bien sûr la tête de la société: c'est sans aucun doute l'allégorie du roi Venceslas, non seulement en tant que représentation animale de tout roi, mais aussi en référence au blason du royaume de Bohême. C'est lui qui convoque le « conseil $^{55}$ » et qui le dirige en accordant la parole aux divers animaux. Malgré le ton critique, sa position n'es nullement contestée. C'est Dieu qui l'a choisi et qui lui a attribué le règne ${ }^{56}$ La seconde place appartient à l'aigle, un invité particulier et un grand seigneur puissant sur son territoire. Il arrive avec sa suite d'oiseaux et le lion l'accueille avec une grande révérence: de façon exceptionnelle par rapport aux autres animaux, il l'embrasse et l'appelle «mon ami». Toutefois, l'aigle est lié au roi lion par un lien de vassalité puisque ce puissant oiseau est prêt à lui apporter l'aide militaire (auxilium) et le conseil (consilium) ${ }^{57}$. Avec ces mentions et le fait que le blason de la Moravie était une aigle, on peut

54. Sur les événements historiques, voir F. M. BARTOš, Čechy v době Husově (13781415), Prague 1947; J. SPěváčer, Václav IV. 1361-1419, Prague, 1986 ; R. Novotv̛́, «Spor

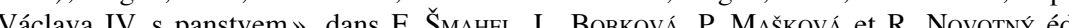
Vactava IV. š p̌rtún Lucemburkové. Ceská koruna uprostred Evropy, Prague, 2012, p. 664-674. Sur la vie de Smil

55. Le terme ne désigne pas ici l'institution du conseil royal mais les animaux exerçant la fonction de conseiller. Pour l'ancrage institutionnel de l'œuvre, voir infra.

56. Cette notion émerge plusieurs fois dans le texte, majoritairement comme l'avertissement du roi censé être responsable devant Dieu, auquel il doit s'apprêter à répondre au jour du Jugement Dernier, et qui peut même le faire chuter: Nová rada, passim.

57. Nová rada, v. 121-122, p. 20 
conclure avec certitude que l'aigle allégorique symbolise le margrave de Moravie, peut-être directement Jošt, patron de l'Union des seigneurs ${ }^{58}$.

L'identification sociale des autres conseillers animaux est considérablement plus difficile, et il serait douteux de se servir de l'héraldique pour trouver des personnes concrètes. On pourrait penser a priori que les oiseaux sont les membres de l'entourage de l'aigle et que les quadrupèdes sont les «gens»du lion. Cependant, plusieurs oiseaux (vautour, coq de bruyère ou chouette) représentent les officiers et mignons du roi Venceslas: cette distinction zoologique ne joue donc aucun rôle dans l'imagination littéraire de Smil Flaška. On ne retrouve que peu de mentions sur le statut des animaux, mais on pourrait penser que l'ordre d'apparition suit une succession hiérarchique indispensable dans un cadre de rencontre de la majesté royale avec l'ensemble du corps politique. De fait, les premiers conseillers se suivent en accord avec leur position sociale. L'aigle prend la parole en premier et son discours est le plus long ${ }^{59}$. Le léopard, qui occupe la place principale dans le conseil royal, reçoit ensuite la parole: il s'agit donc de l'homme le plus important de la scène politique de Bohême après le roi $^{60}$. La parole est ensuite accordée au faucon, car il se tient au plus près du lion $^{61}$. Sur l'appel du roi, c'est l'ours qui parle en quatrième, mais son avis est étonnamment mauvais au regard de sa position privilégiée ${ }^{62}$. Néanmoins l'hypothèse selon laquelle l'ordre de parole reflète le rang dans le royaume est démentie à peu près au milieu du poème avec l'apparition de l'âne, qui représente les couches non-privilégiées ${ }^{63}$ : en effet, d'autres animaux plus importants suivent, par exemple le lynx qui est sans doute noble ${ }^{64}$. Il faut donc renoncer à la reconstruction de la hiérarchie sociale des animaux selon leur ordre dans le poème. Après les premiers, qui sont indéniablement les personnages les plus importants, l'ordre est rompu et les animaux prennent la parole assez librement et sans respecter strictement le protocole.

Le léopard, qui est à la tête du conseil royal, expose l'idéal du roi mais définit également le conseil royal et la sélection des conseillers. Sa conception du bon conseiller correspond parfaitement aux prétentions

58. Cf. J. Skutil, «Heraldické alegorie skladby Orel a král i Nové rady Smila Flašky z Pardubic a z Rychemburka», dans K. MứLLER éd., Sborník př́spěvkĭ ze IV. Setkání genealogù a heraldikㄴ, Ostrava, 1992, p. 113-117.

59. Nová rada, v. 17-55 et 80-370, p. 17-18 et 19-27.

60. Ibid., v. 373-538, p. 28-32. Dans le Nouveau conseil, le léopard ne joue pas du tout un rôle négatif comme on le voit ailleurs dans la littérature médiévale : M. PASTOUREAU, «Quel est le roi des animaux ?», dans Le Monde animal et ses représentations au Moyen Âge (Xle$X V^{e}$ siècles), Toulouse, 1984, p. 135-137; il fait au contraire partie des meilleurs conseillers et personnages du poème.

61. Nová rada, v. 539-542, p. 32.

62. Ibid., v. 573-596, p. 33-34.

63. Ibid., v. 995-1052, p. 46-48

64. Ibid., v. 1483-1518, p. 61-62. de la haute noblesse tchèque depuis l'avènement des Luxembourg ${ }^{65}$. Le léopard est donc un porte-parole de la puissante élite aristocratique (les kniežata de Smil Flaška), qui aspire à participer de manière privilégiée au gouvernement du pays, à la présence au conseil royal et aux offices clés du royaume. Le faucon insiste sur la défense des sujets par le roi et sur sa clémence ${ }^{66}$. La grue, qui est proche du roi et dont les conseils sont très appréciés, se montre soucieuse des offices royaux, surtout dans les régions où les offices judiciaires s'achètent pour le profit qu'on peut en tirer ${ }^{67}$. La cigogne confirme les mots de la grue et accuse des juges régionaux de la justice criminelle (poprávci) de violence et de méfaits. Elle demande que les mauvais officiers soient remplacés par ceux qui «savent gouverner ${ }^{68}$ ». Le chat loue ces paroles et ajoute qu'il faut avoir des espions au service des juges régionaux ${ }^{69}$ : ses soucis font penser que le chat représente une petite noblesse régionale, qui faisait carrière dans les offices locaux de moindre importance ${ }^{70}$. Les revendications qui consistent à réserver le conseil roya aux nobles tchèques, à maintenir la justice dans le pays et l'accès des nobles aux offices de juges régionaux, définissent les articles principaux de l'accusation portée contre le roi par l'Union des seigneurs. Ces animaux doivent donc être interprétés comme des membres de la haute noblesse (le chat semble être un client de la cigogne), qui s'appuient sur le programme de l'Union.

L'opposition à ce parti est formée par des «animaux de forêt», allégories des mauvais officiers et des favoris du roi Venceslas ${ }^{71}$. Le porteparole de cet ensemble est le renard, soutenu par la martre et la loutre. Il s'attaque aux grands seigneurs qui veulent d'après lui dominer le roi par le biais du conseil. Ces nombreux «petits » sont en revanche prêts à bien servir le roi et à le renseigner secrètement sur ce qu'ils ont découvert à la cour afin qu'il puisse régner lui-même ${ }^{72}$. Aussi le loup insiste-t-il sur le pouvoir absolu du roi et propose-t-il d'aller chercher des biens convenables et de les

65. À côté des qualités générales (la loyauté, la sagesse et les vertus morales), le léopard fait de l'origine noble provenant du pays une condition essentielle ; il exclut les étrangers et les non-nobles et demande que le nombre des conseillers soit très restreint : Nová rada, v. 446-456 et 477-494, p. 30-31. On voit les mêmes revendications de la noblesse tchèque envers le roi, dans les négociations de 1310 avec Jean l'Aveugle, et dans les conflits suivants entre le roi et l'opposition aristocratique : J. SPĚvÁČEK, Jan Lucemburský a jeho doba, Prague, 1994.

66. Nová rada, v. 539-572, p. 32-33.

67. Ibid., v. 643-692, p. 36-37.

68. Ibid., v. 1251-1308, p. 54-56

69. Ibid., v. 1309-1324, p. 56.

70. Sur la petite noblesse avant la révolution hussite, voir J. M. KLASSEN, The Nobility and the Making of the Hussite Revolution, New York, 1978; M. PoLívKA, Mikuláš z Husi a nižš́ šlechta v počátcích husitské revoluce, Prague, 1982.

71. M. NejEdLÝ, «L'idéal du roi en Bohême...», p. 255-256.

72. Nová rada, v. 1379-1412, p. 58-59. 
exproprier pour le roi grâce aux machinations judiciaires ${ }^{73}$ : il incarne de la sorte les officiers judiciaires royaux ${ }^{74}$. Le vautour offre au roi la même stratégie de gain injuste par des machinations du droit d'échute et de la tutelle des orphelins ${ }^{75}$. D'autres représentants de ce groupe (l'ours, l'oie, le coq de bruyère, la chouette, etc.) soulignent une faute bien connue de Venceslas IV: l'indifférence à gouverner, qui est selon les seigneurs suscitée et encouragée par son entourage intime ${ }^{76}$. Tous ces animaux sont dépeints de façon particulièrement disgracieuse afin de critiquer explicitement les officiers royaux et les familiers du roi issus de la petite noblesse et de la bourgeoisie aisée.

Mais le poème présente aussi un miroir plus complexe de la cour. Ce sont d'abord les chevaliers qui accentuent l'importance de la représentation de la majesté royale: le paon disserte sur le luxe des vêtements du roi ${ }^{77}$, le cheval sur les festivités et les tournois ${ }^{78}$, tandis que le milan et la buse soulignent l'importance de l'éducation chevaleresque ${ }^{79}$. D'autres aspects de la vie de cour - la curiosité intellectuelle (négative, tendant vers l'alchimie) et la musique (positive, utile au repos) - sont traités par le singe et le rossignol $^{80}$. Le chien sert comme garde du corps du roi ${ }^{81}$ : ce service était effectué par la petite noblesse en tant que de vassaux du roi et c'est ainsi qu'il faut comprendre la figure du chien.

Un autre domaine thématisé est l'armée royale. L'étourneau souligne son pouvoir et le considère comme le plus grand trésor du roi ${ }^{82}$. Le lynx ajoute son savoir-faire en accentuant la discipline dans le champ de bataille ${ }^{83}$. Ces deux conseillers représentent la noblesse militaire et les capitaines des troupes royales. L'importance de la gestion des biens est soulignée par la corneille qui déconseille au roi de partir en voyage et de laisser ses terres sans surveillance: la recommandation plaît aux pies, au moineau et au bruant ${ }^{84}$. Le cerf s'oppose à la guerre: son souci de la paix et de la punition des malfaiteurs concorde avec le soin de l'économie des possessions. On

73. Ibid., v. 699-742, p. 37-38.

74. J. B. C APEK, «Vznik a funkce Nové rady...», p. 20.

75. Nová rada, v. 743-776, p. 39.

76. Ibid., v. 573-598, p. 33-34, v. $977-994$, p. 46, v. $1325-1334$, p. 56-57, v. 1359-1378, p. $57-58$

Ibid., v. 833-860, p. 41-42

78. Ibid., v. 861-904, p. 42-43; cf. M. NEJEDLÝ, «Roi étranger ou roi diplomate ? Jean l'Aveugle au miroir des sources tchèques», Prague Papers on the History of International Relations, 8 (2012), p. 31 .

79. Nová rada, v. 1413-1426, p. 59.

80. Ibid., v. $1551-1573$, p. $63-64$, v. $1683-1722$, p. $68-69$

81. Ibid., v. $1335-1358$, p. 57

82. Ibid., v. 1457-1482, p. 60-61.

83. Ibid., v. 1483-1512, p. 61-62.

84. Ibid., v. 1158-1212, p. 52-53. peut supposer que ces derniers animaux incarnent une noblesse foncière qui s'occupe de ses domaines et de ses revenus.

La dernière couche sociale discernable est représentée par l'âne. Il hésite à se prononcer car ni lui, ni ses oncles n'ont jamais été invités au conseil où «seuls les seigneurs délibèrent». Finalement, il décrit le gouvernement du roi comme un travail difficile, sans lequel n'existent ni les biens, ni l'honneur ${ }^{85}$. L'âne appartient clairement à la couche sociale non noble. Sa remarque sur ses oncles (au lieu de son père) peut signaler le manque de lignage ${ }^{86}$. Son vocabulaire abonde en termes de travail et de sujétion. Issu de la couche des laboratores, l'âne est bien conscient de sa position inférieure et sa participation au conseil est perçue comme inhabituelle et exceptionnelle.

La reconstruction de la composition sociale du Nouveau conseil va de pair avec la question de l'institution politique décrite par l'auteur, qui n'a pas été posée jusqu'alors dans l'historiographie tchèque. À côté du petit conseil royal, deux institutions politiques du royaume de Bohême pourraient correspondre à cette assemblée d'animaux, la «Cour de pays» (zemský soud) et «l'Assemblée de pays» (zemský sněm). La première était la Cour judiciaire suprême du royaume, comparable à un parlement. Elle se réunissait régulièrement quatre fois par an pour juger les causes des nobles et des biens libres. Bien que la cour fût formellement présidée par le roi, cette institution judiciaire était entièrement contrôlée par l'aristocratie et fonctionnait comme le principal lieu de rassemblement de la noblesse tchèque ${ }^{87}$. La Cour n'est donc pas la réunion littéraire de Smil Flaška. L'Assemblée de pays lui correspond en revanche davantage. Cette institution, à l'époque précédant la révolution hussite, est malheureusement très peu connue: pour la période allant de 1378 à 1404, on trouve trace de seulement quatre Assemblées. Son rôle paraît très restreint, d'autant que les réunions se déroulaient sans doute en même temps que celles de la Cour de pays. Grâce aux mandats royaux convoquant ces Assemblées, on peut en connaître la composition sociale. Parmi les participants convoqués figure toujours la haute noblesse, mais pas toujours la petite noblesse et les députés des villes. L'Église est omise à chaque fois, ce qui explique bien l'absence des ecclésiastiques dans le poème. Il est possible aussi que les quelques mandats adressés aux députés des villes représentent des exceptions, si la plupart des Assemblées coïncidaient chronologiquement et par leur composition noble avec les réunions de la Cour de pays. La convocation de l'Assemblée de 1394, promulguée par l'Union des seigneurs, s'adressait

85. Ibid., v. 995-1052, p. 46-48.

86. Sur la terminologie et la définition de la noblesse en Bohême médiévale, voir J. MACEK, Ceská středověká šlechta, Prague, 1997.

87. Cf. Z. HLeDíKOVÁ, J. JANDÁK et J. DoBeš, Dějiny správy v českých zemích od počátkù státu do současnosti, Prague, 2007, p. 43-74. 
ainsi aux barones, nobiles, milites, clientes atque terrigene, c'est-à-dire aux différents niveaux de la noblesse ${ }^{88}$ : la bourgeoisie ne figurait donc pas dans la conception que se faisaient les seigneurs de l'Union de la société politique.

La société décrite dans l'assemblée de Smil Flaška comprend la haute noblesse majoritairement attachée aux idées de l'Union des seigneurs, les officiers royaux et les familiers du roi, la société de cour, la noblesse militaire avec les vassaux royaux, et la noblesse foncière, grande et petite. Le poème ne met donc pas en scène la composition idéale de la société politique telle qu'elle était conçue par les seigneurs de l'Union, mais reflète une situation réelle avec ses différents accès au pouvoir, par la naissance, mais aussi par la propriété ou par le service et les liens étroits avec le roi. Le représentant de la couche des laboratores n'a pour fonction que de renforcer le droit des seigneurs de siéger dans le conseil royal, puisqu'il s'exclut luimême, avouant son incapacité intellectuelle (et physique), nécessaire au conseiller du roi, et se déclarant satisfait de sa position de travailleur. Le Nouveau conseil correspond bien au dualisme du pouvoir que l'on perçoit sous le règne de Venceslas IV : le roi, sa «noblesse de cour» et ses officiers, d'une part, la noblesse de pays avec, à sa tête, les maisons aristocratiques puissantes, d'autre part. L'Assemblée de pays qui sert de cadre au poème est conçue par Smil Flaška comme le lieu de la consultation du roi avec la noblesse, mais sans pouvoir significatif - un pouvoir qu'elle ne gagnera qu'après la révolution hussite sous la forme d'un forum des États ${ }^{89}$. C'est le conseil royal et les offices clés aussi bien que régionaux, toujours tenus par le roi et d'importance capitale pour le gouvernement du royaume, qui sont mis en question par la noblesse et revendiqués par celle-ci dans le poème; la Cour de pays est laissée de côté car elle est déjà aux mains de la noblesse.

La littérature politique dans la Bohême des Luxembourg était dominée par une approche éthique traduisant une vision de la fonction royale clairement définie: le roi doit conduire ses sujets vers le salut. Dans ce but, le roi doit être pieux et sage et il doit s'entourer de sages personnes qui lui soient utiles ${ }^{90}$. À la cour de Charles IV, la sagesse occupait une position très importante parmi les vertus princières. Cette image a influencé toute la littérature politique et nous avons pu démontrer, à travers plusieurs exemples, que cet idéal du roi sage se retrouve dans les miroirs de prince

88. Cf. J. Mezník, «Český zemský sněm za Václava IV.», dans ID., Tvár stárnoucího středověku, Brno, 2008, p. 68-77; S. Russocki, Protoparlamentaryzm Czech do poczatku XV wieku, Varsovie, 1973

89. Cf. J. Mezník, «Vývoj a systém stavovské reprezentace v českých zemích v pozdním středověku», dans ID., Tvár stárnoucího středověku..., p. 105-116.

90. Cf. R. Antonín, Ideální panovník českého středověku. Kulturně-historická skica z dějin středověkého myšlení, Prague, 2013, p. 338-345 et p. 357-364. rédigés en Bohême au cours du XIV ${ }^{\mathrm{e}}$ siècle. De même, l'analyse du Nouveau conseil de Smil Flaška de Pardubice montre, d'une part, une forte influence de l'idéologie de Charles IV et, d'autre part, l'apparition de nouvelles tendances liées à un accroissement des ambitions politiques de l'aristocratie tchèque au cours du règne de Venceslas $\mathrm{IV}$, un règne moins réussi qui fait suite au règne vigoureux de son père. La noblesse souligne la nécessité pour un bon roi de s'entourer de sages conseillers afin d'apparaître la plus apte à jouer ce rôle et essayer ainsi de légitimer ses prétentions à participer au pouvoir.

Jan VoJTí̌́EK - Université Charles de Prague, Faculté des Lettres, Département de l'histoire tchèque (ÚČ́ FF); Université Paris-Sorbonne.

Václav žŮREK - Centrum medievistických studií AV ČR (Centre d'études médiévales), Prague.

Entre idéal et polémique. La littérature politique dans la Bohême des Luxembourg

Au cours du XIV siècle, la littérature politique connaît en Bohême une croissance importante: comme partout ailleurs en Europe occidentale, se développe une littérature engagée en langue vernaculaire. Cette tendance est ici analysee a travers le genre des miroirs des princes selon deux lignes de questionnement. La première partie traite du rôle que joue l'image du roi sage (rex sapiens) dans l'idéologie et la représentation de l'empereur Charles IV de Luxembourg. La seconde partie s'intéresse à la sociéte politique en Bohême telle qu'elle est reflétée dans le poème Nouveau conseil de Smil Flaška de Pardubice, qui se prononce de façon polémique sur règne de son fils Venceslas IV.

allégorie - Bohême - littérature politique - maison de Luxembourg - miroirs des princes - société politique

Between Ideal and Polemic. Political Literature in Luxembourg Bohemia

Political literature flowered and progressed considerably in Fourteenthcentury Bohemia: as well as in the rest of Western Europe, this literature reflected political engagement and was written in vernacular. This tendency is analysed on the basis of the genre of the mirrors for princes in two main directions. The first part deals with the role played by the image of the wise king (rex sapiens) in the ideology and self-presentation of the Emperor Charles IV of Luxembourg. The second part focuses on the political society of Bohemia reflected in the poem New Council by Smil Flaška of Pardubice, which is highly polemical and critical of the reign of his son Wenceslaus IV. allegory - Bohemia - house of Luxembourg - mirrors for princes - politica literature - political society 
